\title{
Pathophysiology: Missing link in Basic-Clinical Meshing in Medical Curriculum in India
}

\author{
Marya, R.K.
}

\begin{abstract}
Pathophysiology is a modern integrative biomedical science that is concerned with the mechanisms responsible for the initiation, development, and treatment of pathological processes in humans. Pathophysiology helps bridge the gap between teaching basic medical sciences and clinical medicine. To illustrate the integrative role of pathophysiology in the medical curriculum, an example of CHF is provided. Chinese and Eastern European countries were pioneers in the recognition of pathophysiology as an independent discipline. In Western Europe, USA and Canada, medical schools do not have independent departments of pathophysiology, though the importance of the discipline of pathophysiology in medical curriculum is well recognized. On the other hand, the subject of pathophysiology is totally neglected in Indian medical schools. In the regulations of $\mathrm{MCl}$ for undergraduate medical education or in undergraduate curriculum of AlIMS (New Delhi), one fails to find any mention of pathophysiology.
\end{abstract}

\section{Introduction}

During the last 100 years, the curriculum of medical schools in India has not changed. Most schools continue to have a fairly rigid division between the first two years of training, which covers the basic sciences, and the final three years, in which students are totally immersed in clinical care. During the first two years, the students are taught anatomy, physiology, biochemistry, pathology, microbiology and pharmacology. Students mug up the basic sciences just to pass the examinations. They have no idea how this knowledge is going to be applied in clinical practice. During the clinical training, the students are taught aetiology, symptoms and signs of a disorder followed by discussion of its treatment. The basic sciences are not brought into the clinical discussion.

\section{Discipline of Pathophysiology}

During the last few decades, medical teachers have felt the necessity of basic-clinical integration.

Department of Physiology, Faculty of Medicine, AIMST University, BEDONG, 08100, Kedah, Malaysia.

Email: rkumarmarya@yahoo.com
However, their desire of basic-clinical integration has usually involved closely coordinated seminars by clinicians and basic scientists to discuss clinical disorders. The side-by-side basic-clinical teaching does not really constitute integration. The key to true basic-clinical integration is pathophysiology.

In many people's minds, the terms "pathogenesis" "pathology" and "pathophysiology" are somewhat ambiguous. Pathogenesis deals with the cellular events during the development of a disease. Pathology deals with the structural defects in a tissue or an organ. In contrast, pathophysiology refers to the functional changes associated with or resulting from a disease or injury. Pathophysiology is a modern integrative biomedical science that is concerned with the mechanisms responsible for the initiation, development, and treatment of pathological processes in humans (Hanacek, 2018).

Pathophysiology, a course for second-year students intends to help bridge the gap between basic science and clinical medicine. The example of pathophysiology of chronic congestive heart failure can be used to illustrate the integrative role of pathophysiology in the medical curriculum.

DOI: http://doi.org/10.4038/seajme.v12i1.43

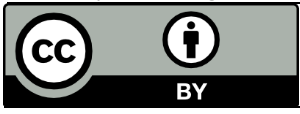

56

(C) SEAJME. This is an Open Access article distributed under the terms of the Creative Commons Attribution License (http://creativecommons.org/licenses/by/4.0/), which permits unrestricted use, distribution, and reproduction in any medium, provided the original author and source are credited

Vol. 12, no. 1, 2018


Pathophysiology of chronic congestive heart failure

Whatever the basic problem, chronic congestive heart failure produces a depression of Frank-Starling Curve. A number of natural compensatory mechanisms are activated so as to improve the cardiac output and maintain normal perfusion of the vital organs, despite depressed state of myocardium. These mechanisms include:

- Frank-Starling mechanism

- Increased adrenergic discharge

A decrease in cardiac output by a failing heart can be compensated by an increase in enddiastolic volume. Retention of salt and water through hormonal mechanisms increases the blood volume and therefore helps to increase ed-diastolic volume of the ventricle. However there is a limit to which this mechanism can help. A large increase in EDV leads to pulmonary congestion and pulmonary oedema.

Increased adrenergic discharge to the heart improves stroke volume at any given EDV. In the failing heart, depressed cardiac output is sensed by high pressure bar receptors located in the carotid sinus and aortic arch, leading to a reflex increase in adrenergic discharge to the heart and blood vessels. In the heart, increased adrenergic discharge improves the cardiac output by increasing the heart rate as well as stroke volume. Increased adrenergic discharge to the blood vessels results in selective vasoconstriction, leading to redistribution of cardiac output. Thus on the basis of pathophysiological changes, various symptoms and signs of CHF can be easily understood (Figure1). Moreover, the use of digitalis and diuretics in the treatment of chronic CHF becomes a rational approach.

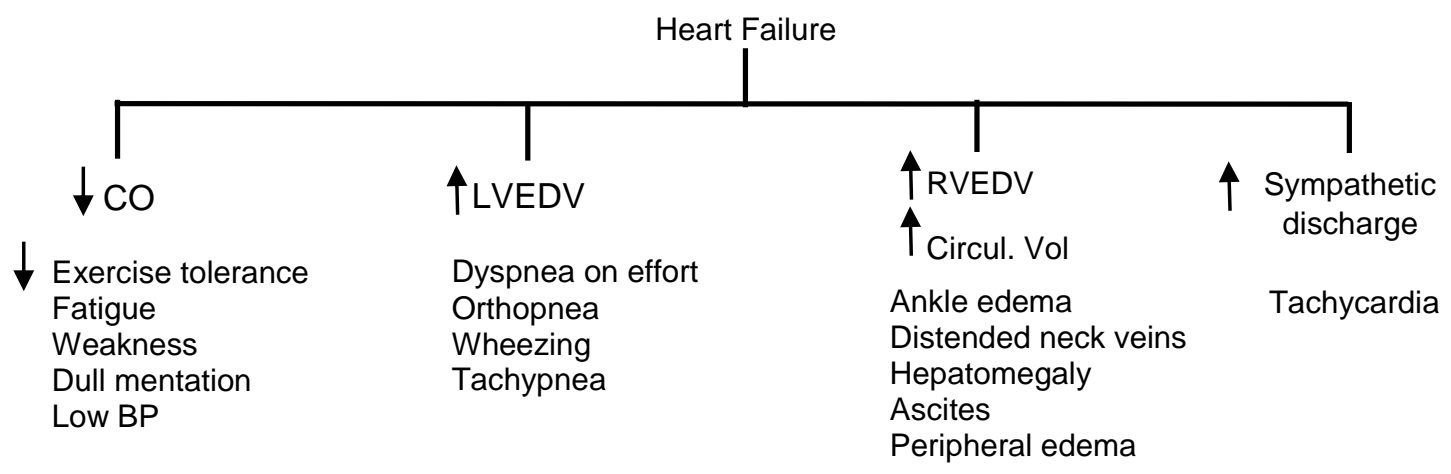

Figure 1: Pathophysiological basis of symptoms and signs of congestive heart failure (CO: Cardiac output; LVEDV: Left ventricular end-diastolic volume; RVEDV: Right ventricular end-diastolic volume)

For advancement of the discipline, an International Society for Pathophysiology and a journal "Pathophysiology" have been launched. Medical educationists now recognize the necessity and importance of the subject of pathophysiology in undergraduate medical curriculum. Integrative pathophysiological teaching/learning increases the student's ability to obtain and retain theoretical, practical, and usable knowledge/competence.

Integration leads to a better understanding, retention of knowledge (Monos, 2007). Knowledge of pathophysiology is also helpful in a rational therapeutic approach to a clinical disorder.

\section{Pathophysiology: missing link in basic-clinical meshing in medical curriculum in India}

Although, pathophysiology belongs to core subjects of undergraduate medical education, the way is is taught varies in different countries. Chinese and Eastern European countries were pioneers in the recognition of pathophysiologyas an independent discipline. Most of the medical schools of these countries have pathophysiology as an independent department (Monos, 2007).

In Western Europe, USA and Canada, medical schools do not have independent departments of pathophysiology though the importance of the discipline of pathophysiology is well 
recognized. In medical curriculum of each medical school, pathophysiology constitutes an important component of preclinical curriculum. For example, in Harvard medical school, pathophysiology is presented throughout the second year in a course organized around organ systems (Harvard Medical School, 2015). On the other hand, the subject of pathophysiology is totally neglected in Indian medical schools. In the regulations of Medical Council of India (MCI) for undergraduate medical education or in undergraduate curriculum of the All India Institute of Medical Sciences (AlIMS), New Delhi, one fails to find any mention of pathophysiology (MCI, 2012; AlIMS, 2005).

\section{Conclusion}

In line with the world wide trends, teaching of pathophysiology should be incorporated in the undergraduate medical curriculum of all the medical schools in India. Moreover, whole curricula need to be reviewed in view of recent innovations in medical education such as problem based learning, self-directed learning and integration at horizontal and vertical level in real sense.

\section{References}

Hanacek, J. (2018) Introduction to Study of Pathophysiologyeng.jfmed.uniba.sk/fileadmin/u ser...v.../04Introduction_to_PF.ppt

Harvard Medical School (2015) https://hms.harvard.edu/.../Design\%20Team\%2 oSummaries\%2011.13.15\%20Final.pd

Monos, E. (2007) History of international society for pathophysiology, Pathophysiology, 14, 2, pp. 123-126.

Medical Council of India (MCl) (1997) Regulations on graduate medical education, New Delhi: Medical Council of India, [Online] Available at http://www.mciindia.org/..../Revised_GME_201 2 pdf.

Syllabus MBBS at the AlIMS (2005). Available at:. http://www.aiims.edu/aiims/academic/aiimssyllabus/Syllabus\%20-\%20MBBS 